\title{
Dynamical evolution of comets from high-eccentricity trans-Neptunian orbits to near-Earth space
}

\author{
Vacheslav V. Emel'yanenko \\ Department of Computational and Celestial Mechanics, South Ural University, Lenina 76 , \\ Chelyabinsk 454080, Russia \\ email: vvemel@math.susu.ac.ru
}

\begin{abstract}
The contribution of high-eccentricity trans-Neptunian objects to the observed populations of both short-period and long-period comets is estimated. About $10^{10}$ objects with a radius $R>0.7 \mathrm{~km}$ in orbits with perihelion distances $28<q<35.5 \mathrm{AU}$ and semimajor axes $60<a<1000 \mathrm{AU}$ are the main source of Jupiter-family comets. If the population of higheccentricity trans-Neptunian objects formed about $4.5 \mathrm{Gyr}$ ago, the mean near-parabolic flux produced by these objects is on the order of 0.3-1.0 $\mathrm{AU}^{-1} \mathrm{yr}^{-1}$ for comets with $R>0.7 \mathrm{~km}$.
\end{abstract}

Keywords. celestial mechanics, comets: general, Kuiper Belt, Oort Cloud

\section{Introduction}

The population of trans-Neptunian objects (TNOs) in high-eccentricity orbits is widely accepted as a source of comets in the inner planetary region. There are two ways by which these TNOs can reach near-Earth space. Due to perturbations of Neptune they can move both inwards and outwards. The inner fraction of objects may directly become Neptune-crossing, Uranus-crossing, Saturn-crossing and Jupiter-crossing (KazimirchakPolonskaya 1972), finally appearing as members of the Jupiter-family (JF) comet system. The bodies diffusing into the outer region may reach large distances where galactic and stellar perturbations are able to inject some of them into the planetary region, creating a flux of near-parabolic bodies.

The basic idea about the origin of JF comets from the flat disc of objects located beyond Neptune can be traced to papers such as (Whipple 1964; Whipple 1972; Fernández 1980b) and others. The detailed study of the relationship between TNOs and JF comets was done in the papers (Levison \& Duncan 1997; Duncan \& Levison 1997). But now the structure of the trans-Neptunian region is known much better than at the time of those papers. Therefore, here we discuss the results of the investigation for the transfer of high-eccentricity TNOs to JF orbits, based on the present orbits of TNOs.

A scattering of bodies from the near-Neptune region to extremely large distances is usually associated with early stages of the Solar system formation (Oort 1950; Fernández 1980a). In combination with galactic and stellar perturbations, it creates the basis for the concept of the Oort cloud (with a dense inner core) (Hills 1981; Duncan, Quinn \& Tremaine 1987) which is a source of observed near-parabolic comets. But it is evident now that high-eccentricity TNOs can penetrate to the Oort cloud region even at the present epoch. Therefore, we study the process of the dynamical evolution of objects from high-eccentricity trans-Neptunian orbits to near-parabolic orbits with perihelia in the inner planetary region. 
Thus, the aim of this paper is to estimate the contribution of high-eccentricity TNOs to the observed populations of both short-period and long-period comets.

\section{High-eccentricity trans-Neptunian objects as a source of Jupiter-family comets}

The detailed investigation of the process by which high-eccentricity TNOs are transferred to short-period orbits was done by (Emel'yanenko, Asher \& Bailey 2004). This work was based on 7 observed high-eccentricity Neptune-approaching orbits of multiopposition TNOs. Each orbit was cloned 249 times. The resulted 1750 orbits were integrated for the age of the Solar system. The comparison of the results of integrations with the observed distribution of JF comets showed that the best fit took place by restricting to $1200 \mathrm{yr}$ the maximum lifetime of comets after their first entry in the JF region defined by the following conditions: the perihelion distance $q<1.5$ AU, the Tisserand parameter $T>2$. With this restriction, the objects spend $460 \mathrm{yr}$ in the JF region on average.

The calculations gave also that the relative fraction of objects captured per year from high-eccentricity orbits with semimajor axes $60<a<1000 \mathrm{AU}$ and perihelion distances $28<q<35.5$ AU to JF comets with $q<1.5$ AU is $0.2 \times 10^{-10}$. Using this value, the fact that there exist about $90 \mathrm{JF}$ comets with a nuclear radius of $\gtrsim 0.7 \mathrm{~km}$ in the region $q<1.5$ AU (Fernández, Tancredi, Rickman \& Licandro 1999) and the above estimate for the lifetime of JF comets we obtained in the paper (Emel'yanenko, Asher \& Bailey 2004 ) that there are $\sim 10^{10}$ TNOs of cometary size in orbits with $60<a<1000$ AU, $28<q<35.5 \mathrm{AU}$.

\section{High-eccentricity trans-Neptunian objects as a source of near-parabolic comets}

Observed 'new' comets come from orbits with $a>10^{4}$ AU. The structure of the transNeptunian population with such semimajor axes is not known. It is evident that this structure is different from that which can be obtained from the study of observed TNOs because the former has been built by a slow evolution of objects for the age of the Solar system. The present distribution of TNOs can give only a general indication about the original one. Therefore, we study the dynamical evolution of 667 objects with a quite broad distribution of initial orbits for $4.5 \mathrm{Gyr}$. Initial perihelion distances and semimajor axes are distributed uniformly in the range $(25,45)$ AU and $(50,300)$ AU, respectively; initial inclinations $i$ are distributed between 0 and 40 degrees according to a sine law with a peak at 20 degrees. Later, different models of the original high-eccentricity transNeptunian population are tested by weighting initial orbits.

The integration of equations of motion is done by using the symplectic integrator (Emel'yanenko 2002; Emel'yanenko, Asher \& Bailey 2003). We include the perturbations from the four outer planets, with the mass of the inner planets being added to that of the Sun. We take account of Galaxy in the way described earlier (Emel'yanenko 1999). Stellar perturbations are described by the impulse approximation, adopting the distribution of parameters for stars from (Heisler, Tremaine \& Alcock 1987).

310 objects that reached the region $a>1000$ AU were cloned 3 times by small changes of the mean anomaly. Then, in order to better study the near-parabolic population in the planetary region after the long-term evolution, all the objects reaching $q<20 \mathrm{AU}$ later than 1.7 Gyr were cloned 19 times. 


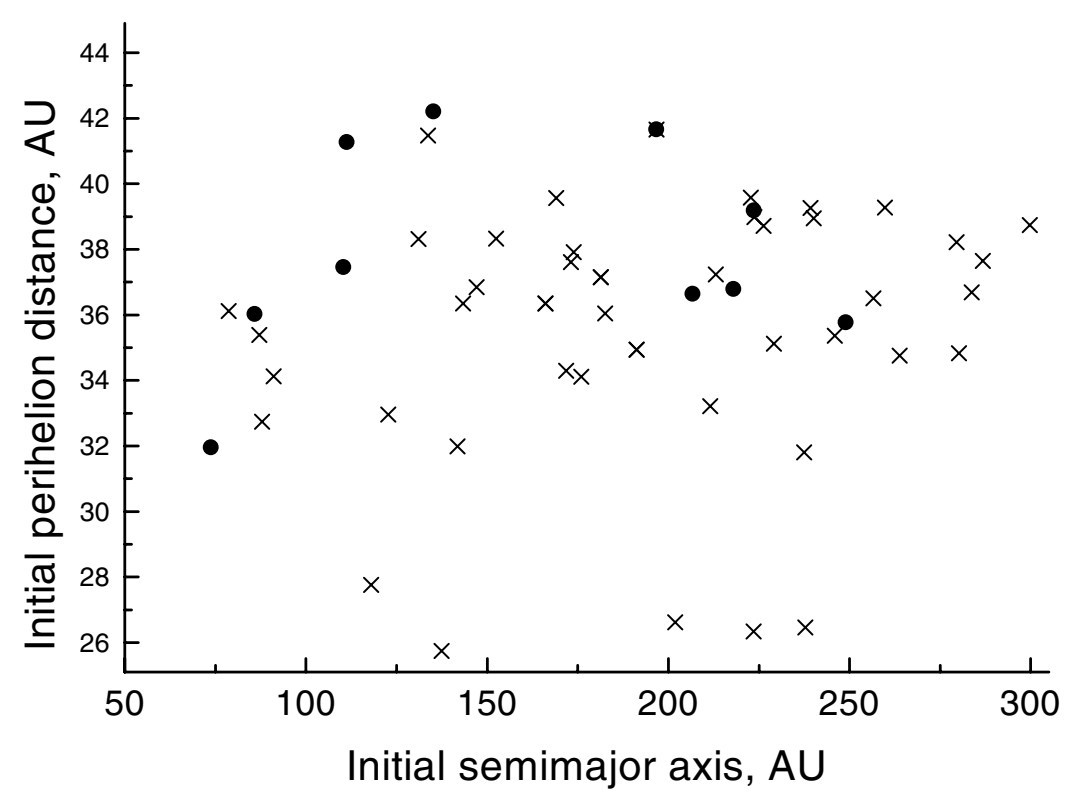

Figure 1. The distribution of initial $a$ and $q$ for objects in orbits with $28<q<35.5$ AU, $60<a<1000 \mathrm{AU}$ (circles) and $q<20 \mathrm{AU}, a>1000 \mathrm{AU}$ (crosses).

In order to quantify the near-parabolic flux produced by TNOs we use the above estimate that there are $\sim 10^{10}$ objects with a radius $R>0.7 \mathrm{~km}$ in the near-Neptune region $60<a<1000 \mathrm{AU}$ and $28<q<35.5 \mathrm{AU}$. In our integration of 667 objects, we have registered 10 objects remaining in this region after $4.5 \mathrm{Gyr}$. On the other hand, 667 initial objects produce on average 1.8 objects reaching the region $q<5 \mathrm{AU}$ with $a>10^{4} \mathrm{AU}$ for the last billion years of our computations. Then we conclude that the mean near-parabolic flux produced by evolved TNOs is equal to $\nu=\frac{1.8 \times 10^{-9} \times 10^{10}}{10 \times 5}=0.36$ $\mathrm{AU}^{-1} \mathrm{yr}^{-1}$.

Even this estimate based on very broad initial conditions shows that former TNOs give a substantial contribution to the near-parabolic flux. But this value depends heavily on the initial distribution of high-eccentricity TNOs. Figure 1 shows the distributions of initial perihelion distances and semimajor axes for objects that remained in the region $28<q<35.5 \mathrm{AU}, 60<a<1000 \mathrm{AU}$ after $4.5 \mathrm{Gyr}$ and objects injected in the region $q<20 \mathrm{AU}, a>10^{3}$ AU later than 3.5 Gyr. The majority of objects surviving in the near-Neptune region have initial $q>36$ AU. If we just assume that high-eccentricity TNOs had mainly orbits with $30<q<36$ AU, $50<a<240$ AU about 4 Gyr ago, then there is only 1 object surviving in the near-Neptune region $60<a<1000 \mathrm{AU}$ and $28<q<35.5 \mathrm{AU}$ (Figure 1). At these initial conditions, we have registered on average 0.46 objects reaching the region $q<5 \mathrm{AU}$ with $a>10^{4}$ AU for the last billion years of our computations. Then the estimated value of $\nu=\frac{0.46 \times 10^{-9} \times 10^{10}}{1 \times 5}=0.92 \mathrm{AU}^{-1} \mathrm{yr}^{-1}$. This is comparable with the observed magnitude. Although the various authors value for the observed near-parabolic flux has a rather wide spread, usually it is estimated as a few comets per year and AU for the size of $\gtrsim 0.7 \mathrm{~km}$ (Bailey \& Stagg 1988; Fernandez \& Gallardo 1999; Wiegert \& Tremaine 1999). 


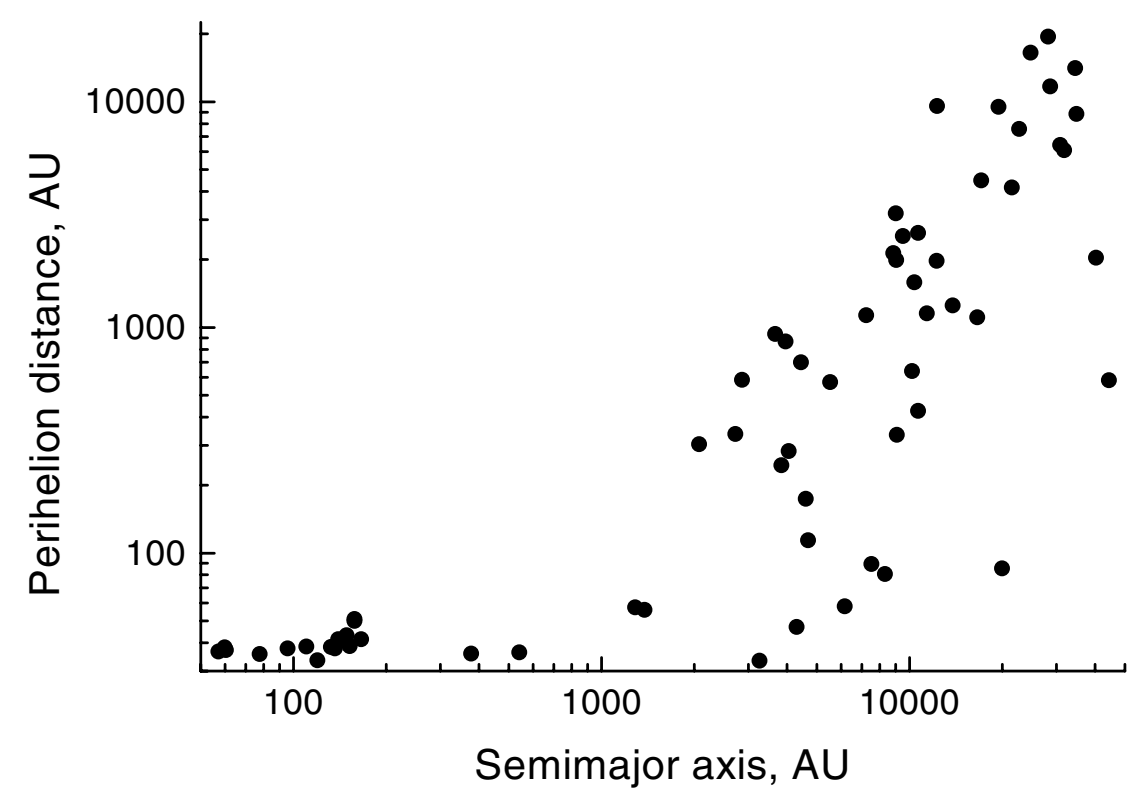

Figure 2. The distribution of $a$ and $q$ for objects with initial $30<q<36$ AU after 4.5 Gyr.

At the initial conditions $30<q<36,50<a<240 \mathrm{AU}$, we have found 65 objects with $q>30$ AU after 4.5 Gyr. Then the estimated number of bodies surviving in the Solar system beyond Neptune after $4.5 \mathrm{Gyr}$ is equal to $\frac{65 \times 10^{10}}{1}=6.5 \times 10^{11}$. The calculated orbital distributions for them are shown in Figures 2 and 3. If the upper limit for $q$ is reduced to $34.5 \mathrm{AU}$, then our computations give on average 0.345 objects reaching the region $q<5 \mathrm{AU}$ with $a>10^{4} \mathrm{AU}$ for the last billion years, 1 object surviving in the nearNeptune region $60<a<1000 \mathrm{AU}$ and $28<q<35.5 \mathrm{AU}$, and 37 objects that have $q>30$ AU after 4.5 Gyr. Then the estimated value of $\nu=\frac{0.345 \times 10^{-9} \times 10^{10}}{1 \times 5}=0.69 \mathrm{AU}^{-1} \mathrm{yr}^{-1}$, and the corresponding number of objects beyond Neptune is equal to $\frac{37 \times 10^{10}}{1}=3.7 \times 10^{11}$. It is necessary to stress that these conditions do not conflict with the distribution of perihelia in the scattered disc model with migrating Neptune (Duncan \& Levison 1997; Gomes 2003).

\section{Conclusions}

The presented computations show that high-eccentricity TNOs give a large contribution to both short-period and long-period comets. $\sim 10^{10}$ objects with $R>0.7 \mathrm{~km}$ in orbits with $28<q<35.5 \mathrm{AU}, 60<a<1000 \mathrm{AU}$ are the dominant source of JF comets. If the population of high-eccentricity TNOs formed about $4.5 \mathrm{Gyr}$ ago, the mean nearparabolic flux produced by these objects is on the order of $0.3-1.0 \mathrm{AU}^{-1} \mathrm{yr}^{-1}$ for comets with $R>0.7 \mathrm{~km}$. 


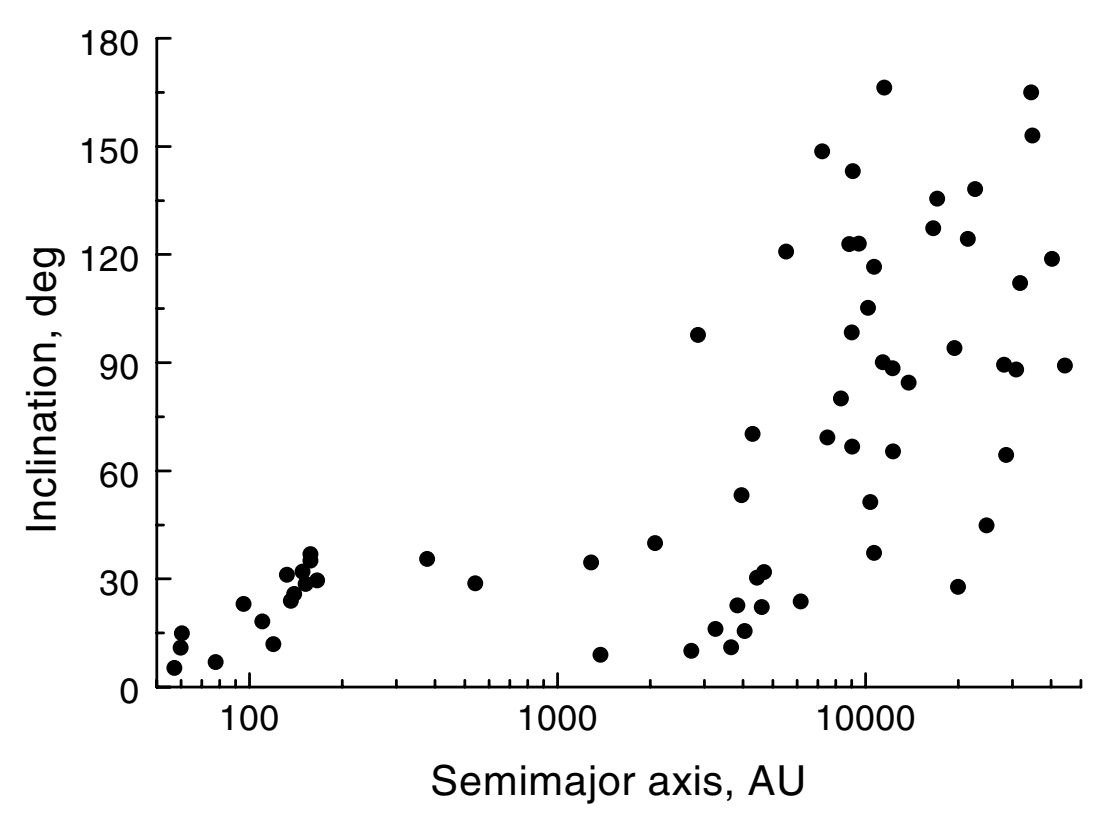

Figure 3. The distribution of $a$ and $i$ for objects with initial $30<q<36$ AU after 4.5 Gyr.

\section{Acknowledgements}

This work was supported by RFBR under grant number 04-02-96042 and INTAS under grant number 00-240. The author thanks an anonymous referee for a careful reading of the manuscript and helpful comments.

\section{References}

Bailey, M.E. \& Stagg, C.R. 1988, Mon. Not. R. Astron. Soc. 218, 1

Duncan, M.J. \& Levison, H.F. 1997, Science 276, 1670

Duncan, M., Quinn, T. \& Tremaine, S. 1987, Astron. J. 94, 1330

Emel'yanenko, V.V. 1999, in: J. Svoren, E.M. Pittich \& H. Rickman (eds.),Evolution and Source Regions of Asteroids and Comets, Proc. IAU Coll. 173 (Tatranska Lomnica: Astron. Inst. Slovak Acad. Sci.), p. 339

Emel'yanenko, V.V. 2002, Celest. Mech. Dyn. Astron. 84, 331

Emel'yanenko, V.V., Asher, D.J. \& Bailey, M.E. 2003, Mon. Not. R. Astron. Soc. 338, 443

Emel'yanenko, V.V., Asher, D.J. \& Bailey, M.E. 2004, Mon. Not. R. Astron. Soc. 350, 161

Fernández, J.A. 1980a, Icarus 42, 406

Fernández, J.A. 1980b, Mon. Not. R. Astron. Soc. 192, 481

Fernández, J.A. \& Gallardo, T. 1999, in: J. Svoren, E.M. Pittich \& H. Rickman (eds.),Evolution and Source Regions of Asteroids and Comets, Proc. IAU Coll. 173 (Tatranska Lomnica: Astron. Inst. Slovak Acad. Sci.), p. 327

Fernández, J.A., Tancredi, G., Rickman, H. \& Licandro, J. 1999, Astron. Astrophys. 352, 327

Gomes, R.S. 2003, Icarus 161, 404

Heisler, J., Tremaine, S. \& Alcock, C. 1987, Icarus 70, 269

Hills, J.G. 1981, Astron. J. 86, 1730 
Kazimirchak-Polonskaya, E.I. 1972, in: G.A. Chebotarev, E.I.Kazimirchak-Polonskaya \& B.G. Marsden (eds.), Proc. IAU Symp. 45, The Motion, Evolution of Orbits, and Origin of Comets (Dordrecht: Reidel) p. 373

Levison, H.F. \& Duncan, M.J. 1997, Icarus 127, 13

Oort, J.H. 1950, Bull. Astron. Inst. Netherlands 11, 91

Whipple, F.L. 1964, Proc. Nat. Acad. Sci. USA 51, 711

Whipple, F.L. 1972, in: G.A. Chebotarev, E.I.Kazimirchak-Polonskaya \& B.G. Marsden (eds.), Proc. IAU Symp. 45, The Motion, Evolution of Orbits, and Origin of Comets (Dordrecht: Reidel) p. 401

Wiegert, P. \& Tremaine, S. 1999, Icarus 137, 84 